\title{
LETTER
}

\section{Power-injectable peripherally inserted central catheters: a step-down access or a real alternative to standard central venous lines?}

\author{
Fernando Godinho Zampieri* \\ See related research by Pittiruti et al., http://ccforum.com/content/16/1/R21
}

The recent article by Pittiruti and colleagues reports important clinical experience with power-injectable peripherally inserted central catheters (P-PICCs) in critically ill patients [1]. Nevertheless, I believe some of the findings deserve clarification.

The authors argue that an advantage of P-PICCs is the possibility to deliver a faster infusion of fluids and/or drugs to critically ill patients. Since P-PICCs were not inserted during emergencies and in severe sepsis, however, the benefit of allowing high flow rates is limited to patients that develop haemodynamic distress after catheter placement. The advantage of allowing infusion of contrast media, although important, is no different from other peripheral noncentral venous devices.

To help understand the role of P-PICCs and to compare complication rates with standard central lines (central venous catheters), it would therefore be interesting to know the severity of illness of patients that eventually received P-PICCs and the percentage of patients that had P-PICCs as their solo central line during their ICU stay. The time interval between admission to the ICU and P-PICC placement would also be interesting data, since the high dwell time reported by the authors suggests that P-PICCs were probably preferentially inserted in patients with prolonged critical illness. If most P-PICCs were inserted in stable chronic patients, the advantage of P-PICCs over conventional peripherally inserted central catheters (PICCs) would be limited in real-life clinical practice.

In addition to comparing P-PICCs with central venous catheters, as suggested by the authors, perhaps further research should also focus on evaluating the role of the P-PICC as a step-down vascular access for stable critically ill patients.

\section{Authors' response}

Mauro Pittiruti and Giancarlo Scoppettuolo

As our study was not a comparative study of the PICC versus the central venous catheter but a pilot retrospective study about the clinical performance of P-PICCs in the ICU, we do not have data comparing the severity of illness between the PICC group and the rest of the patients [1].

Reviewing our population data, however, we found that in most cases - both in adults and in children - PICCs were inserted in the first 48 hours after admission to the ICU (although never as an emergency or in the first 6 hours after admission). In this phase, clinical conditions

*Correspondence: fgzampieri@gmail.com

Intensive Care Unit, Rua Dr. Enéas de Carvalho Aguiar 255, Fifth Floor, Emergency Medicine Discipline, Hospital das Clínicas, University of São Paulo, São Paulo, Brazil may not be completely stable and a sudden need for rapid volume replacement may still occur, so the presence of a central line apt to accept high-flow infusion is reassuring.

On reviewing our data we also found that most adult patients who had a PICC also received other types of central lines (such as dialysis catheters or central venous catheters) during their stay in the ICU. In the majority of paediatric patients and in $30 \%$ of adult patients, the choice of a P-PICC was related to the need for a reliable, safe line for power injection of contrast medium, considering that in many patients (particularly in children) peripheral veins for insertion of a short cannula of appropriate calibre for contrast injection were not available.

These data suggest indirectly that, in our ICU, P-PICCs were not used as an emergency central line - but also were not used as a step-down access. 


\section{Abbreviations}

PICC, peripherally inserted central catheter; P-PICC, power-injectable peripherally inserted central catheter.

\section{Competing interests}

The authors declare that they have no competing interests.

Published: 26 April 2012

\section{Reference}

1. Pittiruti M, Brutti A, Celentano D, Pomponi M, Biasucci DG, Annetta MG, Scoppettuolo G: Clinical experience with power injectable peripherally inserted central catheters in intensive care patients. Crit Care 2012, 16:R21.

\section{doi:10.1186/cc11294}

Cite this article as: Zampieri FG: Power-injectable peripherally inserted central catheters: a step-down access or a real alternative to standard central venous lines? Critical Care 2012, 16:425 